\title{
COMPARAÇÃO ENTRE OS RESULTADOS OBTIDOS NA RECONSTRUÇÃO DO LIGAMENTO CRUZADO ANTERIOR DO JOELHO UTILIZANDO DOIS TIPOS DE ENXERTOS AUTÓLOGOS: TENDÃO PATELAR VERSUS SEMITENDÍNEO E GRÁCIL
}

\author{
COMPARISON BETWEEN THE RESULTS ACHIEVED IN ANTERIOR CRUCIATE \\ LIGAMENT RECONSTRUCTION WITH TWO KINDS OF AUTOLOGOUS GRAFTS: \\ PATELLAR TENDON VERSUS SEMITENDINOUS AND GRACILIS
}

Rene Jorge Abdalla', Diego Antico Monteiro², Leonardo Dias ${ }^{3}$, Dárcio Maurício Correia ${ }^{4}$, Moisés Cohen $^{5}$, Andrea Forgas ${ }^{6}$

\section{RESUMO}

Objetivo: O objetivo deste trabalho é comparar os resultados da artrometria e do exame isocinético entre dois tipos de enxertos autólogos: o terço central do ligamento patelar e o formado pelos tendões dos músculos semitendíneo e grácil, dentro de um mesmo protocolo de reabilitação no sexto mês pós-operatório. Materiais e Métodos: Foram analisados os resultados obtidos dos exames realizados em 63 pacientes divididos em dois grupos. Em um grupo de 30 pacientes, utilizou-se o tendão patelar como enxerto e em outro grupo de 33 pacientes, utilizou-se o tendão dos músculos semitendíneo e grácil. Ambos os enxertos foram fixados da mesma forma, com Endobutton ${ }^{\mathrm{TM}}$ para fixação suspensória no fêmur e um parafuso de interferência bioabsorvível para fixação no túnel tibial. Resultados: A artrometria 30 não apresentou diferença estatística entre os dois grupos observados. Já na avaliação isocinética, constatou-se que o grupo de tendão patelar possui em média maior pico de torque de flexão e maior déficit de extensão e o grupo de flexores possui, em média, melhor relação flexão/extensão e maior déficit de flexão percentual. Não há diferença estatística significante entre os grupos quanto à medida de pico de torque de extensão. Conclusão: portanto, no presente estudo, quando o tendão patelar é utilizado, há maior déficit extensor e quando são utilizados os tendões flexores, há maior déficit flexor.

Descritores - Ligamento cruzado anterior; Joelho; Reconstrução; Artroscopia.

\section{ABSTRACT}

Objective: this study aims to compare the arthrometric and isokinetic examination results from two types of autologous grafts: the central third of the patellar ligament and a graft formed by the tendons of the semitendinosus and gracilis muscles, within the same rehabilitation protocol, six months after the surgery. Methods: the results from examinations carried out on $63 \mathrm{pa}$ tients were analyzed. These patients were divided in two groups: one group of 30 patients who received a patellar tendon graft and another group of 33 patients who received a graft from the tendons of the semitendinosus and gracilis muscles. Both the grafts were attached in the same way, with Endobutton ${ }^{\mathrm{TM}}$ for suspensory fixation to the femur and a bioabsorbable interference screw for fixation in the tibial tunnel. Results: arthrometry 30 did not present any statistical difference between the two study groups. On the other hand, the isokinetic evaluation showed that the patellar tendon group had a larger mean peak torque of flexion and greater extension deficit, while the semitendinosus/gracilis group had a better mean flexion/extension ratio and greater percentage of flexion deficit. There was no statistically significant difference between the groups when measuring peak torque extension. Conclusion: therefore, when the patellar tendon was used, there was greater extensor deficit and, when the semitendinosus/gracilis tendons were used, there was greater flexor deficit.

Keywords - Anterior cruciate ligament; Knee; Reconstruction; Arthroscopy.

\footnotetext{
1 - Doutor em Traumatologia e Ortopedia. Professor do Departamento de Ortopedia e Traumatologia da Unifesp/EPM, São Paulo, SP, Brasil.

2 - Médico estagiário do Centro de Estudos do Centro de Ortopedia e Reabilitação no Esporte - Cecore, São Paulo, SP, Brasil.

3 - Médico Ortopedista do Centro de Estudos do Centro de Ortopedia e Reabilitação no Esporte - Cecore, São Paulo, SP, Brasil.

4 - Médico estagiário do Centro de Estudos do Centro de Ortopedia e Reabilitação no Esporte - Cecore, São Paulo, SP, Brasil.

5 - Professor Livre-Docente. Chefe do Setor de Traumatologia do Esporte do Departamento de Ortopedia e Traumatologia da Unifesp/EPM.

6 - Pós-graduando do Departamento de Ortopedia e Traumatologia da FMUSP.
}

Trabalho realizado no Centro de Estudos do Centro de Ortopedia e Reabilitação no Esporte do Hospital do Coração. №: 08/0005 Correspondência: Andrea Forgas, Rua Abílio Soares, 227, cj. 31. Tel.: 3889-8244. Fax: 3051-8255. E-mail: deaforgas@gmail.com 


\section{INTRODUÇÃO}

Os primeiros relatos de lesão do ligamento cruzado anterior do joelho aparecem na literatura do século IX ${ }^{(1)}$; já os primeiros relatos de reconstrução cirúrgica são do início do século $\mathrm{XX}^{(2)}$, além do de Campbell ${ }^{(3)}$.

Nos últimos 30 anos, foram descritas várias técnicas cirúrgicas para reconstrução ligamentar do LCA, utilizando estruturas vizinhas do joelho. Percorreu-se um grande caminho até retornar à técnica descrita por Campbell ${ }^{(3)}$, em 1939, que utilizou o ligamento patelar. Também no mesmo ano, Macey ${ }^{(4)}$ descreveu a primeira técnica utilizando os tendões flexores - semitendíneo e grácil (ST-G).

Atualmente, com o avanço tecnológico, a reconstrução intra-articular por via artroscópica reduziu a morbidade pós-operatória, porém ainda há controvérsia entre os cirurgiões quanto ao melhor enxerto a ser utilizado.

O objetivo deste trabalho é analisar os resultados do tratamento cirúrgico nas reconstruções do LCA de acordo com o tipo de enxerto escolhido, por meio da avaliação isocinética e da artrometria.

\section{MÉTODO}

Em nosso setor, foram avaliados os resultados de 63 pacientes no sexto mês pós-operatório de cirurgia de reconstrução do LCA, obtidos por meio das avaliações artrométricas $\left(K T-1000^{T M}\right)$ e da avaliação isocinética $\left(C y b e x^{T M}\right)$.

Os critérios de inclusão foram: lesão unilateral do LCA e ausência de antecedentes cirúrgicos ou de patologias em ambos os joelhos e realização do protocolo de reabilitação com a equipe de fisioterapeutas de nosso setor.

Os critérios de exclusão foram: pacientes com idade acima de 60 anos, cirurgias prévias no joelho, lesão bilateral do LCA e realização do protocolo de reabilitação em outro setor.

Os pacientes foram divididos em dois grupos por sorteio de envelope: no primeiro grupo de 30 pacientes, foi utilizado o terço central do tendão patelar (TP) como enxerto e, no segundo grupo de 33 pacientes, os tendões dos músculos flexores semitendíneo e grácil (ST-G).

Em ambos os grupos, a técnica cirúrgica empregada foi a mesma, reconstrução ligamentar intra-articular do LCA por via artroscópica, utilizando para fixação suspensória no fêmur o Endobutton ${ }^{T M}$ e, para fixação no túnel tibial, um parafuso de interferência bioabsorvível. Dos 30 pacientes do grupo do TP, três eram mulheres e 27 homens, com idade variando entre 16 e 37 anos). O lado direito foi acometido em 18 pacientes e o lado esquerdo, em 12.

No grupo do ST-G dos 33 pacientes, cinco eram mulheres e 28 homens, com idade entre 16 e 53 anos. Foram lesionados 13 joelhos do lado esquerdo e 20 do lado direito.
Os 63 pacientes foram submetidos ao mesmo programa de reabilitação, o qual foi dividido em fases. A primeira fase foi iniciada no leito, com mobilização tibiofemoral e patelofemoral, isometria leve, treino de marcha sem apoio com uso de muletas e proteção articular removível (imobilizador). No $10^{\circ}$ dia pós-operatório (PO), segunda fase, é retirada a órtese e autorizada carga parcial. O apoio total e a propriocepção foram liberados no $14^{\circ}$ dia PO. Quanto à amplitude de movimento (ADM), preconizamos ganho progressivo desde a primeira semana pós-operatória, chegando à $\mathrm{ADM}$ total na quarta semana PO. Damos início ao treino de marcha, exercícios de cadeia cinética fechada (CCF), propriocepção avançada e mecanoterapia para o quadril. O fortalecimento global, propriocepção específica para o esporte, avaliação isocinética, equilíbrio e testes funcionais são realizados no sexto mês PO.

Ao término do sexto mês PO, após avaliação isocinética, artrometria e radiografias, o paciente está liberado para as atividades desportivas.

Os pacientes foram submetidos à avaliação artrométrica do joelho com o aparelho $K T 1000^{T M}$, sendo três os testes realizados: teste de tração anterior com forças de 15 , 20 e 30 libras, teste de deslocamento por contração ativa do quadríceps e teste de tração anterior manual máxima. Para a interpretação dos achados artrométricos, foram considerados os valores de diferenças entre o membro acometido e o normal, julgando-se os valores de diferença maiores do que 3mm sugestivos de lesão do LCA; além disso, a positividade de qualquer um dos quatro testes classificava o paciente como portador de lesão do LCA.

A avaliação isocinética iniciou-se com aquecimento prévio de 10 minutos em bicicleta ergométrica, sem carga, com média de 65 a 70 rotações/min., seguida de alongamento de musculatura de membro inferior.

Primeiramente, foi testado o membro sadio e, em seguida, o operado. Nessa avaliação, são mensuradas: força, potência e resistência dos flexores e extensores de joelho, quadríceps e isquiotibiais.

Para designar tais parâmetros, protocolamos o teste da seguinte maneira: cinco repetições a $60 \%$ segundo para força; descanso de um minuto; cinco repetições a $180 \%$ segundo para potência; descanso de um minuto; e 20 repetições a $300^{\circ}$ segundo para resistência, realizando, assim, 30 repetições no total.

Para responder à estatística do trabalho, primeiramente foram descritos os grupos segundo idade, sexo e lado acometido, realizando-se o teste $t$ de Student não pareado ${ }^{(5)}$ para comparar os grupos com relação à idade; foi utilizado o teste exato de Fisher ${ }^{(6)}$ para verificar a associação entre grupo e sexo, e o teste do qui-quadrado de homogeneidade ${ }^{(6)}$ para investigar a associação entre grupo e lado acometido. 
Para comparar os grupos com relação às medidas avaliadas foi realizado o teste $t$ de Student não pareado ${ }^{(5)}$ para cada medida e ilustrados os resultados com o uso de gráficos de medidas com respectivos erros padrões ${ }^{(5)}$. Os testes foram realizados no nível de significância de 5\%.

\section{RESULTADOS}

A média de idade do grupo em que foi utilizado ST-G resultou ser estatisticamente maior do que a encontrada no grupo com TP $(p<0,001)$. De acordo com o sexo, os grupos são estatisticamente homogêneos $(p=0,710)$ e também com relação ao lado acometido $(p=0,102)($ Tabelas 1 e 2).

Tabela 1 - Descrição da idade para cada grupo de procedimento cirúrgico.

\begin{tabular}{llllllll}
\hline Tendão & Média & DP & Mediana & Mínimo & Máximo & $\mathbf{N}$ & $\mathbf{p}$ \\
Patelar & 25,77 & 5,38 & 25,5 & 16 & 37 & 30 & \multirow{2}{0}{, 001} \\
Flexor & 33,55 & 9,80 & 33,0 & 16 & 53 & 33 & \\
\hline Total & 29,84 & 8,85 & 30,0 & 16 & 53 & 63 & \\
\hline
\end{tabular}

Tabela 2 - Porcentagens de sexo e lado acometido para cada grupo cirúrgico.

\begin{tabular}{lllllllll}
\hline \multirow{2}{*}{ Medida } & Categoria & \multicolumn{2}{l}{ Tendão } & \multicolumn{2}{l}{ Total } & p \\
& Patelar & \multicolumn{2}{l}{ Flexor } & & \\
\hline \hline \multirow{2}{*}{ Sexo } & Feminino & 3 & $10,0 \%$ & 5 & $15,2 \%$ & 8 & $12,7 \%$ & \multirow{2}{*}{0,710} \\
& Masculino & 27 & $90,0 \%$ & 28 & $84,8 \%$ & 55 & $87,3 \%$ & \\
\hline \multirow{2}{*}{ Lado } & Direito & 18 & $60,0 \%$ & 13 & $39,4 \%$ & 31 & $49,2 \%$ & \multirow{2}{*}{0,102} \\
& Esquerdo & 12 & $40,0 \%$ & 20 & $60,6 \%$ & 32 & $50,8 \%$ & \\
\hline Total & & 30 & $100 \%$ & 33 & $100 \%$ & 63 & $100 \%$ & \\
\hline
\end{tabular}

Os dois procedimentos apresentam resultados médios distintos com relação às medidas avaliadas. As únicas medidas que não parecem apresentar diferenças entre os dois procedimentos são: a percentagem de pico de torque de extensão (PTEXT\%) e o teste de 30 libras do KT1000 (KT 30) (Figuras 1 e 2).

Pela tabela 3, tem-se que o grupo que utiliza TP possui em média maior de pico de torque em flexão (PTFL\%) ( $p<0,001)$ e maior déficit de extensão ( $<<0,001)$; o

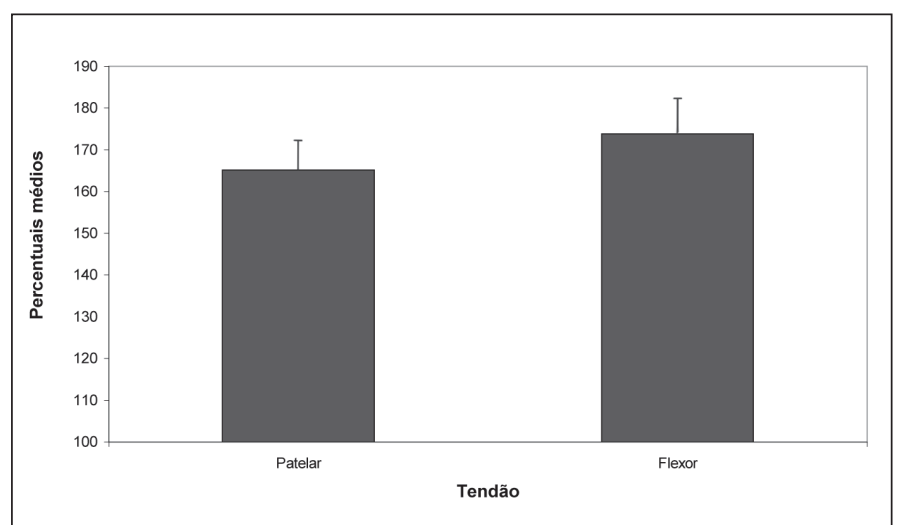

Figura 1 - Valores médios e respectivos erros padrões para a medida PT Ext \%.

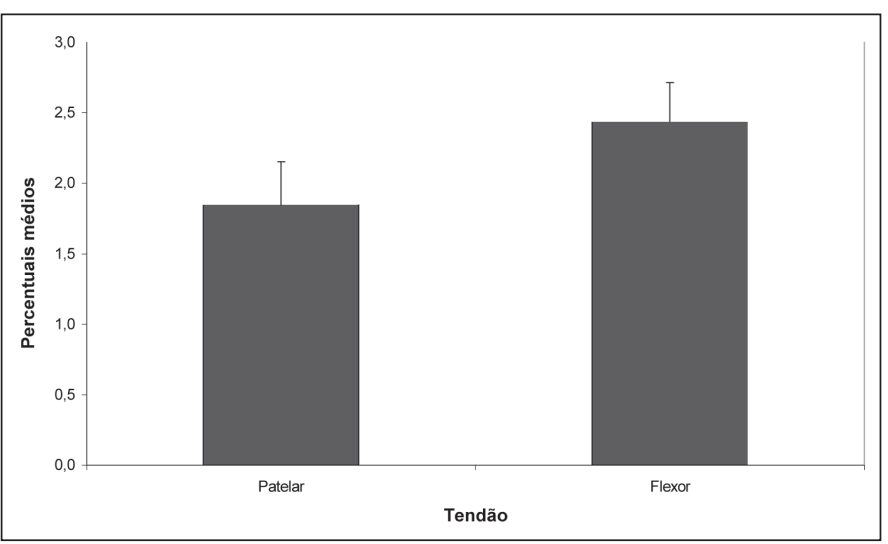

Figura 2 - Valores médios e respectivos erros padrões para a medida KT 30.

Tabela 3 - Descrição das medidas avaliadas para cada grupo e resultado dos testes comparativos.

\begin{tabular}{|c|c|c|c|c|c|c|c|c|c|}
\hline \multirow{2}{*}{ Medida } & \multicolumn{3}{|l|}{ Patelar } & \multicolumn{3}{|l|}{ Flexor } & \multirow{2}{*}{ Valor $t$} & \multirow{2}{*}{ gl } & \multirow[b]{2}{*}{$p$} \\
\hline & Média & DP & $\mathrm{N}$ & Média & DP & $\mathrm{N}$ & & & \\
\hline PTFL\% & 119,18 & 23,82 & 30 & 98,09 & 21,65 & 33 & 3,68 & 61 & $<0,001$ \\
\hline PT Ext. \% & 163,96 & 42,73 & 30 & 172,96 & 50,80 & 33 & $-0,76$ & 61 & 0,448 \\
\hline FL/Ext & 75,42 & 17,79 & 30 & 59,55 & 14,98 & 33 & 3,84 & 61 & $<0,001$ \\
\hline Déficit Flex. \% & $-1,46$ & 13,24 & 30 & 5,78 & 15,06 & 33 & $-2,02$ & 61 & 0,048 \\
\hline Déficit Ext. \% & 29,36 & 13,30 & 30 & 14,74 & 15,29 & 33 & 4,03 & 61 & $<0,001$ \\
\hline KT 30 & 1,87 & 1,43 & 30 & 2,41 & 1,57 & 33 & $-1,43$ & 61 & 0,157 \\
\hline KT Mma. $x$ & 2,18 & 1,80 & 30 & 3,36 & 2,45 & 33 & $-2,191$ & 59 & 0,032 \\
\hline
\end{tabular}

grupo que utiliza ST-G tem, em média, melhor relação entre flexores e extensores (FL/EXT) $(p<0,001)$ e maior déficit flexão percentual $(\mathrm{p}=0,048)$. Não há diferença estatística significante entre os grupos para as medidas de PTEXT\% $\%(p=0,448)$ e KT $30(p=0,157)$.

\section{DISCUSSÃO}

Nos últimos 30 anos, a lesão do LCA teve importantes alterações em sua abordagem terapêutica, retomando a técnica que, em 1939, já utilizava o ligamento patelar como substituto do LCA rompido ${ }^{(3)}$.

Esse regresso às técnicas antigas só foi possível devido aos avanços nos conceitos de anatomia e biomecânica, além da artroscopia com novos instrumentos e das novas técnicas de fixação, o que permite reabilitação mais precoce e melhores resultados. Durante esses 30 anos, vários tipos de enxertos foram utilizados: autólogos, aloenxertos e sintéticos. Na atualidade, os enxertos autólogos de TP e ST-G são preferidos por Wilson e $\operatorname{Scott}^{(7)}$. Em nossa clínica, esses dois tipos de enxertos também são os favoritos, variando a escolha para cada paciente de acordo com o tipo de esporte, tipo de trauma e atividades de vida diária.

Noyes et $a l^{(8)}$ e Corry et al ${ }^{(9)}$ observaram em estudos, que os enxertos de $14 \mathrm{~mm}$ de largura de TP (osso-enxerto-osso) necessitam de força maior para rompe-se do que os outros substitutos do LCA testados separadamente. No entanto, os enxertos de ST-G, quando combinados 
ou quádruplos, passam a ser semelhantes ou mais resistentes que o de TP de $10 \mathrm{~mm}$. Steiner et al ${ }^{(10)}$ estudaram em cadáveres a carga de resistência à lesão dos enxertos: ST-G 4500 N, TP 2646 e LCA intacto 1725 N.

Marder et $a l^{(11)}$ e Aglietti et al ${ }^{(12)}$ realizaram estudos em pacientes com reconstrução do LCA, comparando os dois tipos de enxertos, ST-G e TP, utilizando a mesma técnica de fixação, ou seja, fixação suspensória no fêmur e parafuso de interferência na tíbia para ambos; demonstraram que a anteriorização da tíbia não foi significativa em ambos os grupos, assim como os sintomas. Esses achados correspondem aos nossos; encontramos valores estatisticamente equivalentes para os resultados do artrômetro 30 de ST-G e TP.

Laxdal et $a l^{(13)}$ e Moisala et al ${ }^{(14)}$ também consideram que não há diferença entre os dois grupos quanto à lassidão. Assim como Iorio et al ${ }^{(15)}$ e Ejerhed et $a l^{(16)}$, que não encontram diferenças significativas na artrometria.

Albrecht et $a l^{(17)}$ relataram que os testes funcionais e isocinéticos são usados para avaliar joelhos após cirurgia de reconstrução ligamentar, com o objetivo de estimar a recuperação da força muscular e a relação agonista/antagonista. Concluíram que o membro não operado é boa referência para o estudo isocinético, obtendo resultados com simetria de $95 \%$ entre os membros. Essas características citadas justificam o uso da avaliação isocinética em nosso estudo.

Huston e Woytys ${ }^{(18)}$ observaram que o tempo de recuperação da força muscular é maior nos pacientes com lesão do LCA quando comparado com o de outras lesões.

Quanto à avaliação dos déficits dos isquiotibiais e quadríceps no PO, encontramos publicações com resultados semelhantes aos verificados neste estudo. Coombs e Cochrane ${ }^{(19)}$ estudaram a recuperação da força muscular dos flexores do joelho após a reconstrução do LCA

\section{REFERÊNCIAS}

1. Stark J. Two cases of ruptured ligaments of the knee joint. Edinburgh Med Surg. 1850;74:267-71.

2. Hey-Groves EW. Operation for the repair of the crucial ligaments. Lancet. 1917;2:674-5.

3. Campbell WC. Reconstruction of the ligaments of the knee. Am J Surg. 1939;43:473-80.

4. Macey BH. A new operative procedure for repair of ruptured cruciate ligaments of the knee joint. Surg Gynaecol Obstet. 1939;69:108-9.

5. Bussab WO, Morettin PA. Estatística básica. 4a ed. São Paulo: Atual; 1987

6. Agresti A. Categorical data analysis. 2nd ed. New York: Wiley; 1990.

7. Wilson $A B$, Scott WN. Ligamento cruzado anterior. In: Tria AJ, editor. Lesões ligamentares do joelho. Rio de Janiero: Revinter; 2002.p.159-84.

8. Noyes FR, Butler DL, Grood ES, Zernicke RF, Hefzy MS. Biomechanical analysis of human ligament grafts used in knee ligament repairs and reconstrcutions. J Bone Joint Surg Am. 1984; 66(3):344-52.

9. Corry IS, Webb JM, Clingeleffer AJ, Pinczewski LA. Arthroscopic reconstruction of the anterior cruciate ligament: a comparison of patellar tendon autograft and four-strand hamstring tendon autograft. Am J Sports Med. 1999;27(4):444-54.

10. Steiner ME, Hecker AT, Brown, CH Jr, Hayes WC. Anterior cruciate ligament graft fixation: comparison of hamstring and patellar tendon grafts. Am J Sports Med. 1994;22(2):240-7.

11. Marder RA, Raskind JR, Carroll M. Prospective evaluation of arthroscopycally assisted anterior cruciate ligament reconstruction: patellar tendon versus semitendinosus and gracilis tendons. Am J Sports Med. 1991;19(5):478-84.

12. Aglietti P, Buzzi R, Zaccherotti G, De Biase P. Patellar tendon versus doubled semitendinosus and gracilis tendons for anterior cruciate ligament reconstruction. Am J Sports Med. 1994;22(2):211-8. com ST-G; os resultados mostraram que há déficit de força dos flexores do joelho operado.

Assim como Carter e Edinger ${ }^{(20)}$, nossos resultados também demonstram déficit de força dos músculos quadríceps e flexores após seis meses de cirurgia reparadora do LCA com TP e ST-G; a maioria dos pacientes não apresentava força muscular normal ao final de seis meses de PO.

Feller e Webster ${ }^{(21)}$ também encontraram maiores déficits de extensão no grupo de tendão patelar, com maiores déficits de pico de torque na avaliação com cerca de quatro e oito meses $\mathrm{PO}$, mas não posteriormente. Também encontraram maiores déficits de flexão no grupo ST-G em oito a 24 meses de acompanhamento.

A literatura também mostra alguns estudos conflitantes. Terreri et $a l^{(22)}$ estudaram 18 atletas com média de 21,6 anos (16-32 anos) para avaliar a desempenho após a reconstrução do LCA com TP. Esses autores não encontraram diferença significativa entre os joelhos lesados e os não lesados. A média da relação flexão/extensão em $60^{\circ} \%$ segundo no joelho lesado foi de $60 \%$ e no não lesado, de $57 \%$. Portanto, com o aumento da velocidade, aumentam também os déficits, devido à melhora da desempenho dos flexores, que não é acompanhada pelos extensores.

\section{CONCLUSÃO}

O estudo demonstrou que há semelhança entre ambos os enxertos utilizados. A artrometria apresenta resultados equivalentes. Quanto ao teste isocinético, apesar de haver algumas diferenças em curto prazo, descritas previamente neste trabalho, tais resultados não são confirmados em longo prazo, de acordo com a literatura pesquisada. E não alteram o resultado cirúrgico.

Assim, a escolha do enxerto permanece a critério do cirurgião.

13. Laxdal G, Sernert N, Ejerhed L, Karlsson J, Kartus JT. A prospective comparison of bonepatellar tendon-bone and haminstring tendon grafts for anterior cruciate ligament reconstruction in male patients. Knee Surg Sports Traumatol Arthrosc. 2007;15(2):115-25.

14. Moisala AS, Järvelä T, Kannus T, Järvinen M. Muscle streghth evaluations after ACL reconstrcution. Int J Sports Med. 2007;28(10):868-72.

15. Iorio R, Ciardini R, Russo A, Conteduca F, Ferretti A. Reconstruction of the anterior cruciate ligament with the tendons of the semitendinosus and gracilis doubled: a comparison with reconstruction using the patellar tendon. Chir Organi Mov. 2005;90(4):379-86.

16. Ejerhed L, Kartus J, Sernert N, Köhler K, Karlsson J. Patellar tendon or semitendinosus tendon autografts for anterior cruciate ligament reconstruction?: a prospective randomized study with a two-year follow-up. Am J Sports Med. 2003;31(1):19-25.

17. Albrecht M, Baron R, Petschnig. The relationship between isokinetic quadriceps strength test and hop tests for distance and one-legged vertical jump test following anterior cruciate ligament reconstruction. J Orthop Sports Phys Ther. 1998;28(1):23-31.

18. Huston LJ, Wojtys EM. Neuromuscular performance characteristics in elite female athletes. Am J Sports Med. 1996;24(4):427-36.

19. Coombs $R$, Cochrane T. Knee flexor strength following anterior cruciate ligament reconstruction with the semitendinosus and gracilis tendons. Int J Sports Med. 2001;22(8):618-22.

20. Carter TR, Edinger S. Isokinetic evaluation of anterior cruciate ligament reconstruction: hamstring versus patellar tendon. Arthroscopy. 1999; 15(2):169-72.

21. Feller JA, Webster KE. A randomized comparison of patellar tendon and hamstring tendon anterior cruciate ligament reconstruction. Am J Sports Med. 2003;31(4):564-73.

22. Terreri M, Ambrosio MA, Pedrinelli A, Albuquerque RFM, Andrusaitis F, Greve JMD, et al. Avaliação isocinética do equilíbrio flexo-extensor do joelho nos atletas com ruptura total do ligamento cruzado anterior. Rev Hosp Clin Fac Med São Paulo 1999; 54(2):35-8. 